\title{
VALORACIÓN FUNCIONAL DE LA VISIÓN EN NIÑOS CON DEFICIENCIA VISUAL CEREBRAL
}

\author{
Carlos Manuel Santos Plaza \\ Centro de Recursos Educativos de la ONCE en Madrid \\ carlosantos@telefonica.net
}

Fecha de Recepción: 4 Abril 2018

Fecha de Admisión: 10 Abril 2018

\section{RESUMEN}

La deficiencia visual cerebral es la principal alteración en la función visual en niños en los países desarrollados. Se trata de una afectación distinta, que no está situada en el órgano principal del sentido visual, sino que se produce en los canales o en los centros de interpretación de la información visual. En consecuencia, las repercusiones funcionales son también diferentes y mucho más difíciles de comprender, evaluar e intervenir. No es de extrañar que sea una de las áreas con más líneas de investigación en baja visión en la comunidad internacional. Es necesario revisar cómo se proporcionan los servicios a estas personas con nuevos programas de rehabilitación, modelos de atención multidisciplinarios y una completa evaluación de las funciones visuales. La cuestión fundamental a resolver es cómo afecta el daño en el cerebro a la visión. Cuando el sistema visual está dañado en los centros de procesamiento visual, un amplio rango de efectos se manifiesta y cada caso muestra una combinación de rasgos, muchas veces aparentemente cambiantes y contradictorios. Sólo con una cuidadosa valoración se puede llegar a obtener una aproximación a la comprensión de cómo ve, proporcionando la pauta que debe guiar la intervención para intentar optimizar la eficiencia visual. El modelo de valoración que se presenta considera necesario actuar de forma sistemática a través de entrevistas a los padres y profesores, que son los que mejor conocen los comportamientos visuales del niño, observaciones en el contexto natural y evaluaciones directas de las tareas visuales más significativas.

Palabras clave: deficiencia visual cerebral; niños; valoración funcional de la visión

\section{ABSTRACT}

Functional vision assessment in children with cerebral visual impairment.

Cerebral visual impairment is the main alteration of the visual function in children in developed countries. It is a different affectation, which is not located in the main organ of the visual sense, but is produced in the channels or in the centers of interpretation of the visual information. Thus, the 


\section{VALORACIÓN FUNCIONAL DE LA VISIÓN EN NIÑOS CON DEFICIENCIA VISUAL CEREBRAL}

functional repercussions are also different and much more difficult to understand, evaluate and intervene, It is not surprising that it's one of the areas with more lines of research in low vision in the international community. It is necessary to review how services are provided to these people with new rehabilitation programs, multidisciplinary care models and a complete evaluation of visual functions.

In children with cerebral visual impairment, the main question to be solved is how the damage in the brain affects vision. When the visual system is damaged in the visual processing points, a wide range of effects are manifested and each one shows a combination of particular features which are apparently changing and contradictory. Only with a careful assessment, it is possible to get an approximation to the understanding of how the child sees, providing the guideline which should lead the intervention to try to optimize the visual efficiency. The assessment model introduced, considers that it is necessary to act systematically through interviews with parents and teachers who are the ones who better know the child's visual behaviours, observations in the natural context, and direct evaluations of the most important visual tasks.

Keywords: cerebral visual impairment; CVI; children; functional vision assessment

\section{INTRODUCCIÓN.}

Se puede definir el concepto de discapacidad visual como una disminución visual significativa, pérdida de visión que imposibilita o dificulta de manera importante el acceso a la información y/o causa deficiencias en alguna de las habilidades adaptativas necesarias para la plena integración en la sociedad.

La prevalencia de la deficiencia visual en niños varía desde aproximadamente $0.3 / 1,000$ niños en países más ricos hasta 1.2/1,000 niños en las regiones y países más pobres (Gilbert, 2001). Las enfermedades hereditarias tienen prevalencias similares en todas los países, pero en Canadá, Estados Unidos y Europa se están tratando con éxito las cataratas congénitas y la retinopatía del prematuro, dejando paso a la ceguera cerebral causada por encefalopatías relacionadas con la mayor supervivencia neonatal (G⿳亠丷厂 - Ormaechea y Oyarzabal, 2011).

La deficiencia visual cerebral, DVC, es la principal causa de discapacidad visual bilateral en los niños en países occidentales (Ferell, 2000; Good, Jan, Burden, Skoczenski y Candy 2001; Rahi y Cable, 2003). Aproximadamente el $30 \%-40 \%$ de los niños con deficiencia visual tienen DVC. A su vez se estima que la DVC afecta al $20 \%$ - $40 \%$ de los niños con lesiones cerebrales (Goodrich, 2011). Esta alta incidencia está relacionada con la reducción de la tasa de mortalidad de los bebés prematuros y de muchos niños con graves problemas médicos. Estos niños tienen un riesgo mucho más alto de desarrollar alteraciones neurológicas y deficiencias visuales que los niños nacidos a término.

En la DVC, la afectación no se da en la estructura del ojo o en el nervio óptico sino en los centros de procesamiento visual y en las vías visuales del cerebro (Jan y Groenveld, 1993). La etiología, por orden de incidencia, incluye Hipoxia, Anomalías congénitas del cerebro, Infecciones (meningitis, encefalitis y síndrome de Reye), Traumatismos, Prematuridad y Hemorragia cerebral.

Todavía no hay un acuerdo general con respecto al término más adecuado para describir el daño del cerebro en la función visual. En un reciente análisis (Sakki et al, 2017) se reseña que la terminología con mayor prevalencia en los estudios publicados entre 1979 y 2016, con un $43 \%$ de los mismos, fue la deficiencia visual cortical (Cortical Visual Impairment, CVI), siendo su uso mayoritario en América del Norte (67\% de los artículos). A poca distancia, con un $41 \%$ de los artículos, se encuentra el uso de deficiencia visual cerebral (Cerebral Visual Impairment, CVI), siendo este usado principalmente en Europa. El uso de otras terminología como ceguera cortical, ceguera cerebral se usó sólo en un 16\% de los artículos, y en la mayoría de los casos anteriores al año 2000. 
Aquí se ha decidido usar deficiencia visual cerebral, DVC, por considerarlo más adecuado, ya que los daños cerebrales no solo se producen en la corteza visual.

Como indican García-Ormaechea y Oyarzabal (2011) hay muy poca bibliografía sobre el pronóstico visual de los niños con deficiencia visual cerebral al ser un diagnóstico reciente en todos los países. Estas autoras citan tres estudios prospectivos en los que se sigue la evolución visual y oculomotora de 67 niños (Hoyt, 2003), 70 niños (Matsuba y Jan, 2006) y 53 niños (Bova y al, 2008), con conclusiones finales bastante parecidas. En general la capacidad visual, referida a agudeza visual y control oculomotor, no mejora en la mitad de estos niños, y sin embargo, mejora mucho en una pequeña proporción, que va del 4\% al $17 \%$. La mejoría se aprecia en una fijación estable, con buen control de los movimientos sacádicos, y agudeza visual superior a 0.3. Siempre evolucionan peor las lesiones cerebrales mas extensas, especialmente las que presentan los niños con parálisis cerebral infantil $(\mathrm{PCI})$ tetraparética.

Hay muy pocos estudios epidemiológicos sobre deficiencia visual severa infantil, el más completo se realizó en el Reino Unido encontrando que la mayor incidencia es durante el primer año de vida (4x 10.000) aunque la muestra alcanza los 16 años. El 48\% del total de los niños tienen lesiones en las vías visuales (Deficiencia Visual Cerebral), 29\% tienen una retinopatía (14\% una distrofia retiniana), y $28 \%$ una neuropatía (13\% una atrofia). $77 \%$ de los niños tienen otras deficiencias asociadas. En la mitad de estos niños no había sospecha familiar de mala visión. Concluyen que hay una asociación importante entre la deficiencia visual y las alteraciones en el neurodesarrollo, (Rahi y Cable 2003).

En un estudio, dirigido por especialistas del Royal Belfast Hospital for Sick Children (Reino Unido), se ha procurado establecer el perfil del deficiente visual en la infancia (Flanagan, N. M., Jackson, A. J. \& Hill, A. E., 2003):

El $32 \%$ mostraba un desarrollo normal.

Un $43 \%$ tenía dificultades graves o retrasos para aprender.

Un $21 \%$ de los niños presentaban solo deficiencia visual.

El 79\% tenían problemas médicos adicionales; de ellos, el más común era parálisis cerebral, con un porcentaje del $33 \%$.

El 9\% de los niños fueron diagnosticados como totalmente ciegos.

En un $45 \%$, se halló un deterioro visual cortical.

Estudios recientes realizados en otros países desarrollados han dado resultados muy similares y aunque se carece de estadísticas completas en España se puede considerar que las cifras son parecidas, y como indican G ${ }^{\mathrm{a}}$ - Ormaechea y Oyarzabal (2011), no hay duda de que la principal patología causante de la deficiencia visual severa en niños es de origen neuroftalmológico y en un cerebro en desarrollo.

Según el área del cerebro afectada presentarán características muy diferenciadas pero existen algunas características generales, comportamientos típicos e indicativos de la presencia de una deficiencia visual cerebral (Langley, 1990; Roman-Lantzy 2008):

Se observa una cara inexpresiva.

Ausencia o respuestas visuales reflejas atípicas.

Realizan movimientos oculares suaves pero sin finalidad.

No utilizan habitualmente la comunicación visual.

Falta de atención y curiosidad visual. La actividad visual espontánea es de duración breve.

La visión tiende a fluctuar al ser influenciada por factores como el cansancio, el ruido, un entorno desconocido, la medicación...

La visión periférica parece ser más funcional que la central. 


\section{VALORACIÓN FUNCIONAL DE LA VISIÓN EN NIÑOS CON DEFICIENCIA VISUAL CEREBRAL}

Perciben y atienden mejor a los objetos en movimiento que a los estáticos.

Ausencia de alcance visual. Cuando alcanzan a ver un objeto vuelven la cabeza.

El aprendizaje visual parece producir cansancio.

Los objetos se ven a distancias cortas.

Los objetos sólo pueden reconocerse en ambientes conocidos.

Las caras pueden no ser reconocidas.

Tienen dificultades para ver objetos o imágenes que están muy juntas, dando el Ilamado efecto "masificación" (crowding).

Parece que son incapaces de combinar la vista con otros sentidos.

La función visual está influida por un control deficiente de la cabeza.

La posición del cuerpo puede influir en el uso de la visión.

Retardo en la respuesta visual.

\section{VALORACIÓN FUNCIONAL DE LA VISIÓN EN NIÑOS CON DEFICIENCIA VISUAL.}

La evaluación funcional de la visión debe ser un proceso de recopilación, valoración e integración de información sobre las características funcionales de la visión, debe abarcar tanto los aspectos relacionados directamente con el funcionamiento de los ojos como los referidos a la eficiencia en el uso de la visión que cada niño consigue. Los primeros se refieren por tanto a aquellos aspectos cuantificables que van a determinar el grado de deficiencia visual (agudeza visual, campo visual, sensibilidad al contraste...). La eficiencia en el funcionamiento visual pretende evaluar las destrezas y habilidades que el niño pone en práctica para acceder a la información, incluye por tanto no solo los aspectos visuales sino también los perceptivos, cognitivos..., valora el uso que la persona en su conjunto hace de su sentido visual. Por lo tanto este tipo de evaluación debe ser realizada por un equipo multiprofesional (Santos, Ramos y Rodríguez, 2013).

En primer lugar se debe realizar una evaluación oftalmológica completa que comprende básicamente la misma exploración habitual, pero con una metodología de trabajo diferenciada para intentar obtener el máximo de resultados significativos. Se evalúa la agudeza visual de lejos y cerca, el campo visual, la sensibilidad al contraste, la visión binocular, la visión del color, la motilidad ocular... Se explora la parte anterior del ojo, biomicroscopía, la tensión ocular y el fondo de ojo.

A continuación el óptico-optometrista debe evaluar los posibles defectos de refracción y realiza la prescripción inicial de las ayudas ópticas, no ópticas y electrónicas. El examen optométrico consta de una parte objetiva, en la que se valora la refracción ocular, donde no es necesaria la respuesta del niño, y otra parte subjetiva, en las que la colaboración y la atención de los niños son imprescindibles.

La valoración propiamente funcional tiene como primer objetivo ayudar a determinar de la forma más exacta posible los datos cuantitativos, estos se refieren especialmente a agudeza visual (lejos y cerca), campo visual y sensibilidad al contraste. Esta información debe complementar los resultados obtenidos en la exploración oftalmológica al intentar obtener respuestas significativas, con la participación de otros especialistas con un perfil más psicopedagógico y en distintas condiciones ambientales más cercanas al niño, que permitan corroborar u obtener nueva información de las capacidades visuales.

En el caso de los niños más pequeños y/o con menor nivel de respuesta una evaluación inicial debe incluir la respuesta a una luz, la respuesta a una amenaza, la respuesta a diferentes objetos, a Ios colores, a dibujos, a personas, a caras (Crossman, 1992).

En recién nacidos, incluidos los niños prematuros, se puede utilizar la Batería de Modelos Leonhardt («ML»), para la primera evaluación visual en bebés y primera infancia (García-Trevijano y al., 2008). 
Para obtener una primera medida de Agudeza visual se puede utilizar el Test de Mirada Preferencial. Esta prueba consiste en la presentación de dos tarjetas o raquetas; una con franjas alternantes blancas y negras y otra de color gris, reflejando ambas la misma cantidad de luz. Las raquetas van disminuyendo la anchura de franja, siendo éstas cada vez más finas y próximas entre sí. El niño tiende a fijar su atención en la banda "rayada" más que en la "lisa" siempre que pueda llegar a percibir la diferencia entre ambas, en caso contrario le parecerán idénticas y recibirán la misma atención. La anchura de franja menor en la que se aprecie preferencia en la mirada indica el grado de visión del niño Esta prueba se ha aplicado con éxito a bebes a partir de un mes, aunque se obtienen los mejores resultados desde los 4 hasta los 9 meses, aproximadamente, a partir de este momento los mecanismos de atención del niño empiezan a ser más complejos. Diferentes estudios han demostrado su utilidad para la valoración binocular de la Agudeza en niños normales, de baja visión y plurideficientes. En estos últimos es necesario realizar adaptaciones de la técnica, y aumentar el tiempo de duración del test, para adaptarlo a las características individuales de cada niño (Oyarzabal, 1996).

Cuando se dispone de un nivel mayor de respuesta se pueden emplear los test L.H., creados también por la Dra. Lea Hyvärinen. Se trata de una batería de tests que utilizan como optotipo cuatro figuras muy sencillas (círculo, cuadrado, manzana/corazón y casa). Se tratan ya de tests baremados de reconocimiento, así que se obtienen medidas de la Agudeza Visual en cualquiera de las notaciones tradicionales (Snellen, fracción o logarítmica). Existen test de Agudeza Visual, tanto de lejos como de cerca, y Sensibilidad al Contraste.

El campo visual se evalúa por medio de diferentes técnicas de confrontación. Lo importante es conseguir centrar la atención del niño hacia un estímulo situado enfrente de sus ojos, y comprobar si percibe visualmente la presencia de otros estímulos que se introducen en diferentes partes del campo.

Tan importantes como los datos cuantitativos es obtener información sobre cómo utiliza la visión y el nivel de desarrollo perceptivo visual. La entrevista con la familia, la información que aportan otros profesionales y el uso de planillas de observación son herramientas imprescindibles si se quiere conocer las estrategias que utiliza, los objetos y personas a los que presta atención visual, las distancias, los colores que más le atraen, la iluminación con la que se encuentra más cómodo, etc.

La tarea del especialista que trabaja con niños con deficiencia visual es determinar donde falla cada alumno a lo largo del continuo del desarrollo visual, y entonces diseñar instrucciones que le enfrenten a las habilidades propias de su edad (Ferrell, 2010). En otras palabras la evaluación del funcionamiento visual debe identificar las tareas visuales que el niño/a no es capaz de realizar o que presentan un desarrollo inferior al que debería tener superado.

En este tipo de evaluaciones hay que ser extremadamente prudente. Como expresa perfectamente Ferrell (2010), un niño con deficiencia visual que no exhibe un comportamiento visual particular puede hacerlo más tarde bajo condiciones diferentes, quizás mas apropiadas, 0 en presencia de la persona adecuada. En consecuencia antes de concluir que un niño no es capaz de realizar una particular habilidad visual, siempre hay que asumir que es posible que pueda hacerlo en otras situaciones.

\section{VALORACIÓN FUNCIONAL DE LA VISIÓN EN NIÑOS CON LESIÓN CEREBRAL.}

En el caso de los niños con deficiencia visual, las valoraciones tienen como objetivo determinar la influencia de los problemas visuales en el desarrollo escolar del estudiante. La pregunta que guía la evaluación es cómo afecta el daño o la disfunción ocular a las habilidades del niño. Por el con- 


\section{VALORACIÓN FUNCIONAL DE LA VISIÓN EN NIÑOS CON DEFICIENCIA VISUAL CEREBRAL}

trario en los niños con DVC la cuestión fundamental a resolver es cómo afecta el daño en el cerebro a la visión (Santos y Ramos, 2013).

El modelo que aquí se propone parte de la detección precoz. El Pediatra debe ser capaz de identificar la existencia de la deficiencia y derivar a los especialistas para realizar la Valoración diagnóstica. Es en esta fase cuando se debe intentar determinar el tipo y grado de la problemática visual del niño. Es muy importante que esta exploración esté en manos de oftalmólogos especializados. La detección temprana de deficiencias visuales es fundamental para poder minimizar sus consecuencias, especialmente en niños con daño cerebral (Fazzi y al, 2007). Como señalan Leonhardt y al.(2012) la evaluación de las funciones visuales forma parte de los exámenes neurológicos y actualmente se incluye en los métodos usados más ampliamente en las evaluaciones de los recién nacidos.

A continuación es necesario que se realice una Valoración funcional que debe plantearse como un proceso continuado cuyo objetivo debe ser identificar las características de la visión de cada niño, valorar la manera de conseguir el máximo desarrollo de sus capacidades y obtener información significativa para desarrollar los programas de intervención.

Cuando el sistema visual está dañado en los centros de procesamiento visual cada caso muestra una combinación de rasgos particulares y muchas veces aparentemente cambiantes y contradictorios. Sólo con una cuidadosa valoración de las conductas visuales del niño se puede llegar a obtener una aproximación a la comprensión de cómo ve, proporcionando la pauta que debe guiar la intervención para intentar optimizar la eficiencia visual.

Para ello es imprescindible actuar de forma sistemática a través de entrevistas, observaciones y evaluaciones directas del niño (Roman-Lantzy, 2008).

\section{Entrevistas con padres, profesores...}

En el caso de los niños con DVC es necesario otorgar una especial importancia a la información que pueden proporcionar los familiares en el protocolo de actuación, ya que los padres son los mejores conocedores del comportamiento visual de sus hijos. Los padres y otros miembros de la familia de los niños con DVC poseen información fundamental acerca de cómo los niños responden visualmente y las formas en que el niño puede mostrar las características concretas de carácter visual y de comportamiento típico de DVC. Es por ello que una entrevista cuidadosamente redactada con un padre o tutor puede ser un método muy adecuado para ser aplicado en la detección de indicadores positivos de la existencia de una deficiencia visual cerebral.

Roman-Lantzy (2008) ha diseñado un Cuestionario para su presentación a los padres formado por 25 preguntas. Cada pregunta se plantea de modo abierto, y según la respuesta proporcionada por los padres o tutores se le asigna una puntuación positiva (la respuesta es sugerente de presencia de DVC), o puntuación negativa (la respuesta no indica DVC) al compararla con una Guía de respuestas, revelando un perfil indicativo de DVC si la mayoría de resultados son positivos.

\section{Observación del niño tanto en el entorno escolar como en el familiar.}

La observación directa y sistemática en el contexto natural de las conductas visuales del niño es fundamental para conocer e intentar comprender la visión de los niños con deficiencia visual cerebral.

Existen algunas planillas de observación que pueden ser de cierta utilidad, aunque es necesario adaptarlas al contexto de cada niño antes de administrarlas. Por ejemplo el Informe-Observación del Proyecto Ivey (Smith-Cote, 1983), diseñado para ayudar a los Educadores de alumnos con plurideficiencia a dejar constancia de sus observaciones sobre comportamiento y respuestas del estudian- 
te. Consta de tres partes en las que se pretende reflejar los Comportamientos relacionados con el Movimiento, el Comportamiento Sensorial y los Comportamientos posturales.

En el caso de los niños con DVC es conveniente observar los comportamientos visuales habituales, la forma en la que usan su visión en sus actividades cotidianas. Hay que fijarse en el grado de atención visual, en su motilidad ocular, en las formas de respuesta a los estímulos visuales, en el uso de los sentidos para extraer información, en la variabilidad de las respuestas según sea el entorno, las estrategias que utiliza, los objetos y personas a los que presta atención visual, las distancias, los colores que más le atraen, la iluminación con la que se encuentra más cómodo, la zona del campo visual más funcional...

\section{Evaluación directa.}

En el caso de los niños con DVC la evaluación de las capacidades visuales estará muy condicionada por los daños neurológicos y por las características típicas del funcionamiento visual de esta población. Las pruebas para medir las funciones visuales son las mismas reseñadas anteriormente, pero obtener una medida de la agudeza Visual, sensibilidad al contraste, campo visual..., resulta en muchas ocasiones una tarea especialmente complicada.

En los niños más pequeños o con menor nivel de respuesta la agudeza visual se intenta con el test de Mirada Preferencial, el campo visual por medio de diferentes técnicas de confrontación, la sensibilidad al contraste con el test Hidding Heidi..., pero cuando la atención visual es mínima no se obtienen resultados fiables.

En las evaluaciones funcionales de la visión en niños con DVC es especialmente importante controlar que el ambiente esté libre al máximo de interferencias sensoriales que puedan influir en las respuestas. Una de las características frecuentes en esta población es que sólo prestan atención a un canal de entrada de información, y normalmente es dominante el auditivo, por lo que cualquier ruido y mucho más si se trata de una conversación puede producir que desconecten totalmente la atención visual.

Antes de pasar cualquier prueba es necesario contactar con el niño, empatizar con él, que el niño se familiarice con el evaluador y éste con sus estrategias comunicativas. En muchas ocasiones es conveniente contar con la colaboración de los padres y/o del profesor o profesional más adecuado para acceder al alumno.

Existen diversas pruebas que pueden aportar información sobre la respuesta del niño en tareas visuales significativas. Es necesario utilizar estos instrumentos como herramientas que permiten obtener una rápida información sobre algunos aspectos especialmente relevantes. Las pruebas que se pueden utilizar en la valoración de la visión de alumnos con deficiencia visual cerebral son:

En los primeros meses se puede utilizar la Batería de Modelos Leonhardt («ML»), para la primera evaluación visual en bebés y primera infancia (García-Trevijano, Leonhardt y al., 2008).

En niños con baja respuesta visual se pueden usar:

La Evaluación de la Visión Funcional del proyecto IVEY (Smith y Cote, 1983). Diseñada para valorar los niveles más bajos de desarrollo visual, evaluando principalmente la conciencia visual y la capacidad motriz visual.

La Evaluación de la Baja Visión del método VAP-CAP (Blanksby, 1993). Elaborada para considerar la capacidad visual y los niveles básicos de atención visual. Se busca obtener información de cuánto puede ver el niño y de cómo se motiva su atención visual.

La Prueba de valoración de la Visión Funcional para personas que No Colaboran con el examinador, PVFNC (Ruf y Torrents, 2008). Diseñada para valorar el resto visual de personas con deficiencia visual y graves trastornos asociados (físicos, psíquicos, sensoriales) que dificultan su colaboración con el examinador. 


\section{VALORACIÓN FUNCIONAL DE LA VISIÓN EN NIÑOS CON DEFICIENCIA VISUAL CEREBRAL}

CVI Range (Roman-Lantzy, 2008). Es un método de evaluación elaborado específicamente para valorar a niños con deficiencia visual cerebral. Se trata de un método diseñado para determinar el grado en que el niño está afectado por las 10 características más relevantes del comportamiento visual de los niños con DVC (preferencia de color; necesidad de movimiento; retardo en la respuesta visual; preferencia de campos visuales; dificultad hacia la complejidad visual; fijación hacia la luz y mirada perdida; dificultades con la visión de lejos; ausencia o respuestas visuales reflejas atípicas; dificultad con estímulos visuales nuevos; ausencia de alcance visual)

\section{CONCLUSIONES}

La deficiencia visual cerebral es la principal alteración en la función visual en niños en los países desarrollados. Se trata de una afectación distinta, que no está situada en el órgano principal del sentido visual, en el ojo, sino que se produce en los canales o en los centros de interpretación de la información visual. En consecuencia, las repercusiones funcionales son también diferentes y mucho más difíciles de comprender, evaluar e intervenir, pero se trata de una deficiencia visual que hay que comenzar a abordar con mayor profesionalidad.

Para ello, es necesario partir de un diagnóstico lo más temprano posible y una valoración funcional de la visión que intente entender cómo afecta el daño en el cerebro a la visión. Para conseguir este difícil propósito se debe estructurar un protocolo que incluya entrevistas con los padres y las personas que más conozcan al niño; observaciones de las conductas visuales en el entorno habitual del niño y evaluaciones directas utilizando instrumentos adecuados.

El resultado final de la valoración debe ser reseñar las claves para iniciar o continuar la intervención con el objetivo de optimizar la funcionalidad visual. El propósito de la intervención para estudiantes con DVC es procurar que usen su visión tanto como sea posible. A través del uso de la visión más sinapsis se desarrollan en las áreas visuales del cerebro. Esencialmente, a los alumnos hay que proporcionarles estímulos a los que sean capaces de mirar.

Por último es importantísimo que los profesionales entiendan que los padres de los niños con DVC necesitan algo más que información. En muchas ocasiones los familiares se encuentran desorientados al no saber cómo pueden ayudar mejor a sus hijos, y en demasiadas ocasiones reciben instrucciones contradictorias. Es necesario hacerles entender que la lesión cerebral es la causante de las conductas visuales atípicas y a veces desconcertantes, y una vez finalizada la valoración intentar explicarles que esos comportamientos tienen su explicación.

\section{REFERENCIAS BILIOGRÁFICAS.}

Blanskby. D.C. (1993). Visual Assesment and Programming. The VAP-CAP Nandbook. Royal Institute for the Blind. Victoria. Australia. Traducción castellano. Evaluación Visual y Programación. Manual VAP-CAP. Córdoba (Argentina): Christoffel Blindenmission.

Bova, S.M. y al. (2008). Recovery of visual functions after early acquired occipital damage. Dev Med \& Child Neurol, 311- 315.

Crossman. H.L (1992). Cortical Visual Impairment presentation, assessment and management. The Royal New South Wales Institute for Deaf and Blind Children. Monograph series- no3. North Rocks. Australia: North Rocks Press.

Fazzi, E., Signorini, S.G., Bova, S.M., La Piana, R., Ondei, P., Bertone, C., y al. (2007). Spectrum of visual disorders in children with cerebral visual impairment. J Child Neurol;22(3): 294-301.

Ferell, K.H. (2000) "Growth and Development of Young Children", in Holbrook, M.L. et al. (eds) Foundations of Education, 2nd edn, Vol. 1, pp. 111-34. New York: American Foundation for the Blind. 
Ferrell, K. A. (2000). Growth and development of young children with visual impairments. En M. C. Holbrook \& A. M. Koenig (Eds.), Foundations of education for children and youths with visual impairment (pp. 111-134). New

York: AFB Press.

Ferrell, K.A. (2010). Visual Development. En Foundations of Low Vision: Clinical and Functional Perspectives, 2nd Edition. Corn, A.L. Y Erin, J.N. Editors. New York. AFB Press

Flanagan, N. M., Jackson, A. J. \& Hill, A. E., (2003). Visual impairment in childhood: insights from a community-based survey. Child Care Health Dev, 29, 493-499.

García-Ormaechea, I. y Oyarzábal, B. (2011). Deficiencia Visual Discapacitante en los niños de nuestro entorno: Etiología, Diagnóstico y Rehabilitación. Acta Estrabológica, 2011.2

García-Trevijano, C.. Leonhardt, M., Oyarzábal, B., Vecilla, I. y Lafuente, M.A.(coord.). (2008).

Construir juntos espacios de esperanza: orientaciones para el profesional de atención temprana a niños con ceguera o deficiencia visual. Madrid: ONCE.

Gilbert, C. y Foster, A. (2001). Childhood Blindness in the context of VISION 2020. Bulletin of the WHO, 79 (3): 227-232.

Good, W.V., Jan, J.E., Burden, S.K., Skoczenski, A. \& Candy, R. (2001) "Recent Advances in Cortical Visual Impairment”, Developmental Medicine and Child Neurology 43(1): 56-60.

Goodrich, G. (2011). Neurologic Vision Impairment: A New Direction for Low Vision Research and Rehabilitation. Paper in The 10th Internacional Conference on Low-Vision, KL VISION 2011. Kuala Lumpur (Malasia).

Hoyt, C.S. (2003). Visual function in the brain- damaged child. Eye., 17, 369- 384.

Jan, J., y Groenveld, M. (1993). Visual behaviors and adaptations associated with cortical and ocular

impairment in children. Journal of visual impairment and blindness (JVIB), 87, 101-105.

Langley, B. (1990). Potencial Assessment of Visual Efficiency. Louisville, Kentucky: American Printing House for the Blind.

Leonhardt, M. y al. (2012). Visual performance in preterm infants with brain injuries compared with low-risk preterm infants. Early Hum Dev. doi:10.1016/j.earlhumdev.2012.02.001

Oyarzabal, B. (1996). Nuestra experiencia con el test de Mirada Preferencial en niños con baja visión y plurideficientes. En: Actas del Congreso Estatal sobre Prestación de Servicios para Personas Ciegas y Deficientes Visuales. Madrid: ONCE

Matsuba, C.A. y Jan, J.E. (2006). Long- term outcome of children with cortical visual impairment. Dev Med \& Child Neuro, 508- 512.

Rahi, J. S. y Cable, N. (2003). Severe visual impairment in children in the UK. The Lancet, Vol 362 , 1359-1365.

Roman-Lantzy, C. (2008). Cortical Visual Impairment: An Approach to Assessment and Intervention. New York: AFB Pres.

Ruf, A. y Torrents, T. (2008). Prueba de valoración de la Visión Funcional para personas que No Colaboran con el examinador (PVFNC). Madrid: ONCE.

Santos, C.M. y Ramos, M.A, y Rodríguez, E. (2013) La deficiencia visual cerebral. En Santos, C.M. (Ed). La discapacidad visual. Implicaciones en el desarrollo. El reto de la inclusión educativa. Madrid. Sanz y Torres.

Santos, C.M., Ramos, M.A, y Rodríguez, E. (2013) Detección y evaluación de las deficiencias visuaIes. En Santos, C.M. (Ed). La discapacidad visual. Implicaciones en el desarrollo. El reto de la inclusión educativa. Madrid. Sanz y Torres.

Smith A.J., Cote K.S. (1983). Proyecto IVEY (Increasing Visual Efficiency). Departamento de 


\section{VALORACIÓN FUNCIONAL DE LA VISIÓN EN NIÑOS CON DEFICIENCIA VISUAL CEREBRAL}

Educación del Estado de Florida. Traducción interna ONCE.

Ferell, K.H. (2000) "Growth and Development of Young Children", in Holbrook, M.L. et al. (eds) Foundations of Education, 2nd edn, Vol. 1, pp. 111-34. New York: American Foundation for the Blind.

Sakki, H., Dale N., Sargent, J., Pérez T. y Bowman R. (2017). Is there consensus in defining childhood cerebral visual impairment? A systematic review of terminology and definitions. British Journal of Ophthalmology 10.1136/bjophthalmol-2017-310694. (In press).

Gilbert, C. y Foster, A. (2001). Childhood Blindness in the context of VISION 2020. Bulletin of the WHO, 79 (3): 227-232.

Good, W.V., Jan, J.E., Burden, S.K., Skoczenski, A. \& Candy, R. (2001) "Recent Advances in Cortical Visual Impairment", Developmental Medicine and Child Neurology 43(1): 56-60.

Rahi JS, Cable N. Severe visual impairment and blindness in the UK. Lancet 2003;362:1359-65. doi:10.1016/S0140-6736(03)14631-4 [Published Online First: 23 October 2003]. 